Original article

\title{
Total tumor load assessed by one-step nucleic acid amplification assay as an intraoperative predictor for non-sentinel lymph node metastasis in breast cancer
}

\author{
Celso Nabais ${ }^{\mathrm{a},}{ }^{*}$, Joana Figueiredo ${ }^{\mathrm{a}}$, Paulina Lopes ${ }^{\mathrm{a}}$, Manuela Martins ${ }^{\mathrm{b}}$, António Araújo ${ }^{\mathrm{a}}$ \\ ${ }^{a}$ Department of Surgery, Centro Hospitalar Lisboa Central, Portugal \\ ${ }^{\mathrm{b}}$ Department of Pathology, Centro Hospitalar Lisboa Central, Portugal
}

\section{A R T I C L E I N F O}

\section{Article history:}

Received 15 August 2016

Received in revised form

1 October 2016

Accepted 19 December 2016

\section{Keywords:}

Breast cancer

One-step nucleic acid amplification

Sentinel lymph node

Non-sentinel lymph node metastasis

Axillary lymph node dissection

Total tumor load

\begin{abstract}
A B S T R A C T
Background: This study aimed to determine the relationship between CK19 mRNA copy number in sentinel lymph nodes (SLN) assessed by one-step nucleic acid amplification (OSNA) technique, and nonsentinel lymph nodes (NSLN) metastization in invasive breast cancer. A model using total tumor load (TTL) obtained by OSNA technique was also constructed to evaluate its predictability.

Methods: We conducted an observational retrospective study including 598 patients with clinically T1T3 and node negative invasive breast cancer. Of the 88 patients with positive SLN, 58 patients fulfill the inclusion criteria.

Results: In the analyzed group $25.86 \%$ had at least one positive NSLN in axillary lymph node dissection. Univariate analysis showed that tumor size, TTL and number of SLN macrometastases were predictive factors for NSLN metastases. In multivariate analysis just the TTL was predictive for positive NSLN (OR 2.67; 95\% CI 1.06-6.70; $\mathrm{P}=0.036$ ). The ROC curve for the model using TTL alone was obtained and an AUC of 0.805 (95\% CI $0.69-0.92)$ was achieved. For TTL $>1.9 \times 10^{5}$ copies/ $\mu$ L we got $73.3 \%$ sensitivity, $74.4 \%$ specificity and $88.9 \%$ negative predictive value to predict NSLN metastases.

Conclusion: When using OSNA technique to evaluate SLN, NSLN metastases can be predicted intraoperatively. This prediction tool could help in decision for axillary lymph node dissection.
\end{abstract}

๑) 2016 Elsevier Ltd. All rights reserved.

\section{Introduction}

Sentinel lymph node (SLN) biopsy have become the standard technique for determining axillary nodal involvement in patients with early-stage breast cancer who are clinically negative.

Identifying the SLN as non-metastatic spares unnecessary axillary lymph node dissection (ALND) and therefore decreases the chances of significant co-morbidities [1].

Intraoperative diagnosis of positive SLN can allow ALND in the same surgical procedure when criteria are present, thus avoiding a second surgery to treat the axilla and decreasing the patient's associated discomfort and institutional costs $[2,3]$.

The one-step nucleic acid amplification (OSNA, Sysmex, Kobe, Japan) assay is a molecular method that measures the quantity of cytokeratin (CK)-19 mRNA (a duct epithelial cell marker that is highly

\footnotetext{
* Corresponding author. Department of Surgery, Centro Hospitalar Lisboa Central, Rua José António Serrano, 1150-199, Lisboa, Portugal.

E-mail address: celso.nabais@gmail.com (C. Nabais).
}

expressed in more than 95\% of breast cancers) in axillary lymph nodes [4]. Cutoff values were defined to classify macrometastases (more than 5000 copies $/ \mu \mathrm{L}$ ), micrometastases $(250-5000$ copies $/ \mu \mathrm{L}$ ) and negative nodes (fewer than 250 copies/ $\mu \mathrm{L}$ ) [5].

Combined analysis of nine studies that compared OSNA with histopathology demonstrated high concordance between both methods (96\%) and reported high sensitivity, specificity and negative predictive value for OSNA [2]. As the OSNA assay is essentially an automated procedure, it has clear advantages in standardization, reproducibility and objectivity.

There is so far no clear consensus on how to approach ALND. Several studies have identified predictors of metastases to nonsentinel lymph nodes (NSLNs), to select patients who can be spared ALND, and different nomograms have been proposed to select patients who would not benefit from ALND.

Recently, the American College of Surgeons Oncology Group (ACOSOG) Z0011 trial defined a select cohort of patients with positive SLNs in whom a complete ALND may be safely omitted [6]. However, many patients still require prediction of non-SLN metastases. 
With the increasing use of OSNA, different groups have started to study the relationship between CK19 mRNA copy number and the NSLN metastization.

Ohi et al. (2012) and Osako et al. (2013) demonstrated that the NSLN macrometastatic rate increased in proportion to CK19 mRNA copy numbers [7,8]; Ohi et al. verified that the CK19 mRNA copy number in SLN is the most important predictive factor of NSLN metastases, and that higher copy numbers are strongly associated with four or more axillary lymph node metastases [7].

Other groups also evaluated the correlation between the total tumor load (TTL) in SLN and additional NSLN metastases [9,10]. Banerjee et al. (2014), in a small subgroup of 45 women who had undergone ALND, found that using the CK19 mRNA copy number alone resulted in an AUC of 0.828, which indicates that OSNA is more useful than nomograms in predicting the risk of NSLN metastasis [2].

This study aimed to determine the relationship between CK19 mRNA copy numbers and subsequent NSLN metastization, and to determine the TTL intraoperatively as a threshold above which metastases are expected, to support surgeons' oncological decisions regarding the need to perform ALND.

\section{Materials and methods}

This observational retrospective study was conducted between October 2010 and December 2014, and initially enrolled 598 women with invasive breast cancers. Inclusion criteria were patients whose disease had been assessed clinically and ultrasonographically as node-negative and at tumor stage cT1-3, and who had undergone intraoperative SLN evaluation by OSNA. We excluded patients who had received neoadjuvant treatment, whose biopsies showed CK19-negative tumors, or those who did not undergo ALND. Of the 598 patients, 88 had positive SLNs. Of these 88, 61 had been analyzed by OSNA, three of whom were excluded because they had not undergone ALND. Finally, 58 valid patients were studied. Data collected included age, tumor size, grade, histological subtype, estrogen and progesterone receptor status, HER2 status, Ki67, lymphovascular invasion, multifocality, total number of SLNs and non-SLNs, type of surgery, the number of positive and negative non-SLNs, and CK19 mRNA copies.

SLNs were detected using radioisotopes and blue dye, and sent for pathological analysis. When macrometastases were found during intraoperative evaluation, patients underwent level II ALNDs. Depending on patient and tumor characteristics, lumpectomies or mastectomies were also performed.

OSNA evaluations were completed for the isolated SLNs. The OSNA assay procedure has already been described in detail [4]. The analysis result included the number of CK19 mRNA copies per $\mu \mathrm{L}$. These copy numbers were used semi-quantitatively to characterize node involvement; those with $<250$ copies/ $\mu \mathrm{L}$ were considered non-metastatic, $250-5000$ copies $/ \mu \mathrm{L}$ as having micrometastases, and $>5000$ copies $/ \mu \mathrm{L}$ as having macrometastases.

NSLNs obtained from ALND were studied after being processed by histopathologic methods. Immunohistochemical staining was not used.

\subsection{Statistical analysis}

Data were evaluated descriptively, with frequencies used for categorical variables and medians for continuous variables. Chisquare and ANOVA tests were used to compare positive and negative NSLNs. We conducted univariate and multivariate analyses, from which non-significant variables $(P>0.05)$ were dropped. Logistic regression was used to assess the capacity of the studied variables to identify positive NSLNs.

The TTL variable was studied using area under the receiver operating characteristic (ROC) curve (AUC), after log transformation to avoid nonlinearities. The statistical analyses were carried out in SPSS 20.0 for Windows and MedCalc 15.8.

\section{Results}

\subsection{Patients' characteristics}

Of the 58 patients with positive SLNs who were analyzed by OSNA in this study, 15 (25.86\%) were found to have positive nodes

Table 1

Patient's characteristics and comparison of negative versus positive NSLN.

\begin{tabular}{|c|c|c|c|}
\hline & Negative NSLN $(n=43)$ & Positive NSLN $(\mathrm{n}=15)$ & $P$ value \\
\hline Age - years $($ mean $\pm S D)$ & $57.10 \pm 11.09$ & $57.77 \pm 12.68$ & 0.717 \\
\hline Histologic type & & & 0.371 \\
\hline Invasive ductal carcinoma & 35 & 12 & \\
\hline Invasive + ductal carcinoma in situ & 3 & 1 & \\
\hline Invasive lobular carcinoma & 5 & 1 & \\
\hline Invasive papillary carcinoma & 0 & 1 & \\
\hline Tumor size (mean in $\mathbf{m m} \pm \mathrm{SD}$ ) & $20.65 \pm 7.06$ & $25.93 \pm 10.79$ & 0.035 \\
\hline Lymphovascular invasion & & & 0.469 \\
\hline Yes & 20 & 8 & \\
\hline No & 20 & 5 & \\
\hline ER & & & 0.440 \\
\hline Positive & 36 & 14 & \\
\hline Negative & 6 & 1 & \\
\hline PR & & & 0.061 \\
\hline Positive & 29 & 14 & \\
\hline Negative & 13 & 1 & \\
\hline HER2 & & & 0.699 \\
\hline Positive & 7 & 2 & \\
\hline Negative & 30 & 12 & \\
\hline SLN number $($ mean \pm SD) & $2.23 \pm 1.15$ & $2.60 \pm 1.06$ & 0.282 \\
\hline SLN macrometastases (mean \pm SD) & $1.19 \pm 0.55$ & $1.80 \pm 0.68$ & 0.001 \\
\hline NSLN resected $($ mean $\pm S D)$ & $11.86 \pm 6.11$ & $12.80 \pm 5.36$ & 0.599 \\
\hline TTL $-\log ($ mean $\pm S D)$ & $4.65 \pm 0.84$ & $5.54 \pm 0.71$ & 0.000 \\
\hline
\end{tabular}

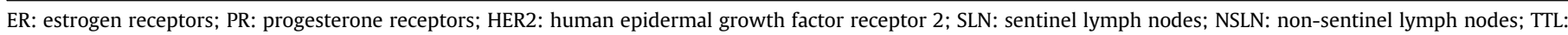
total tumor load. 
Table 2

Univariate and multivariate analyses of NSLN macrometastases.

\begin{tabular}{|c|c|c|c|c|}
\hline & $\begin{array}{l}\text { OR }(95 \% \mathrm{CI}) \\
\text { Univariate }\end{array}$ & $\begin{array}{l}P \\
\text { Univariate }\end{array}$ & $\begin{array}{l}\text { OR }(95 \% \mathrm{CI}) \\
\text { Multivariate }\end{array}$ & $\begin{array}{l}P \\
\text { Multivariate }\end{array}$ \\
\hline Age (years) & $0.99(0.94-1.05)$ & 0.711 & & \\
\hline Tumor size (mm) & $1.08(1.00-1.16)$ & 0.047 & $1.06(0.98-1.15)$ & 0.168 \\
\hline Log TTL (copies/ $\mu \mathrm{L})$ & $3.56(1.57-8.09)$ & 0.002 & $2.67(1.06-6.70)$ & 0.036 \\
\hline SLN macrometastases & $4.64(1.55-13.92)$ & 0.006 & $2.63(0.94-7.36)$ & 0.066 \\
\hline \multicolumn{5}{|l|}{ Histologic type } \\
\hline Invasive ductal carcinoma & 1 & 0.974 & & \\
\hline Invasive + ductal carcinoma in situ & $0.97(0.09-10.26)$ & & & \\
\hline Invasive lobular carcinoma & $0.58(0.06-5.51)$ & & & \\
\hline Invasive papillary carcinoma & - & & & \\
\hline \multicolumn{5}{|l|}{ Histological tumor grade } \\
\hline $\mathrm{I}$ & 1 & 0.069 & & \\
\hline II & $0.15(0.02-1.05)$ & & & \\
\hline III & $1.14(0.18-7.28)$ & & & \\
\hline Lymphovascular invasion (yes vs no) & $1.60(0.45-5.74)$ & 0.471 & & \\
\hline ER (positive vs negative) & $2.33(0.26-21.17)$ & 0.451 & & \\
\hline PR (positive vs negative) & $6.28(0.75-52.90)$ & 0.091 & & \\
\hline HER2 (positive vs negative) & $0.71(0.13-3.94)$ & 0.699 & & \\
\hline Multifocality (yes vs no) & $2.78(0.53-14.48)$ & 0.226 & & \\
\hline
\end{tabular}

ER: estrogen receptors; PR: progesterone receptors; HER2: human epidermal growth factor receptor 2; SLN: sentinel lymph nodes; TTL: total tumor load.

in ALND. The eight patients had a mean age of 56.7 years, mean tumor size of $21.79 \mathrm{~mm}$, mean SLN number of 2.31, and 1.36 macrometastases. The mean TTL was $5.86 \times 10^{5}$ copies/ $\mu \mathrm{L}$. More detailed patients' characteristics, stratified by positive or negative NSLN, are shown in Table 1.

\subsection{Univariate and multivariate analyses}

In univariate analysis, we considered age, tumor size, TTL, SLN macrometastases, histologic type, histological tumor grade, lymphovascular invasion, ER, PR, HER2 and multifocality; and found tumor size, TTL and number of SLN macrometastases to be predictive factors of NSLN metastases $(P<0.05$; Table 2$)$.

In multivariate analysis, TTL was the only independent predictor of NSLN metastases (odds ratio [OR]: 2.67; 95\% confidence interval $[\mathrm{CI}]: 1.06-6.70 ; P=0.036$; Table 2 ). Both tumor size and number of SLN macrometastases were significantly associated with additional NSLN metastases in univariate analysis, but not in multivariate analysis (OR: $1.06 ; 95 \% \mathrm{CI}: 0.98-1-15 ; P=0.168$ and OR: $2.63 ; 95 \%$ CI: 0.94-7.36; $P=0.066$, respectively).

Intraoperative TTL assessment by OSNA was further studied by ROC curve. The AUC of TTL/log TTL was 0.805 (95\% CI 0.69-0.92; $P<0.05$; Fig. 1). We then used the ROC curve to choose a cutoff point for TTL at $\log$ TTL $>5.28$ or TTL $>1.9 \times 10^{5}$ copies $/ \mu \mathrm{L}$, which gave $73.3 \%$ sensitivity, $74.4 \%$ specificity, and $88.9 \%$ negative predictive value for NSLN metastases. In this sample there were 15 patients with NSLN metastases, 11 of whom had TTL $>1.9 \times 10^{5}$ copies $/ \mu \mathrm{L}$.

\section{Discussion}

SLNs are the first axillary nodes to receive lymphatic flow from primary tumors and the most likely to harbor tumor cells. If SLN has no metastases, ALND can be safely avoided [1]. However, if the SLN is positive for macrometastases, ALND is the standard procedure, even though more than half of these patients have no NSLN metastases. In our study, only $25.86 \%$ of patients with positive SLNs had NSLN metastases.

Several prediction models for NSLN metastases in patients with positive SLNs have been developed, but most are based on postoperative histological findings, and are therefore not helpful for intraoperative decision making.

OSNA assay is a molecular-based intraoperative detection method for SLN metastases that has several advantages over frozen sections; it is a fast, standardized technique in which the entire lymph node is examined and provides quantitative results [2].

In this study, univariate analysis showed that tumor size, number of SLN macrometastases and CK19 mRNA copies (TTL) determined by OSNA assay were significantly associated with nonSLN metastases. These results are in line with other studies $[9,10]$. However, in multivariate analysis, TTL was the only variable with a significant association that could be used intraoperatively.

Reportedly, TTL can be used as a predictor for non-SLN metastases $[9,10]$. We studied the prediction model with TTL using the ROC curve, which had good discrimination acuity $(A U C=0.805)$ in this group of patients. At a TTL cutoff of $>1.9 \times 10^{5}$ copies $/ \mu \mathrm{L}$, there was $73.3 \%$ sensitivity and $74.4 \%$ specificity in predicting NSLN metastases. This cutoff was calculated considering a low false positive rate to avoid hypothetically unnecessary ALND in post Z0011 era. Espinosa-Bravo et al. (2013) also reported similar results [9].

After the results of the ACOSOG Z0011 trial were reported, the standard practice of ALND in the presence of positive SLNs was

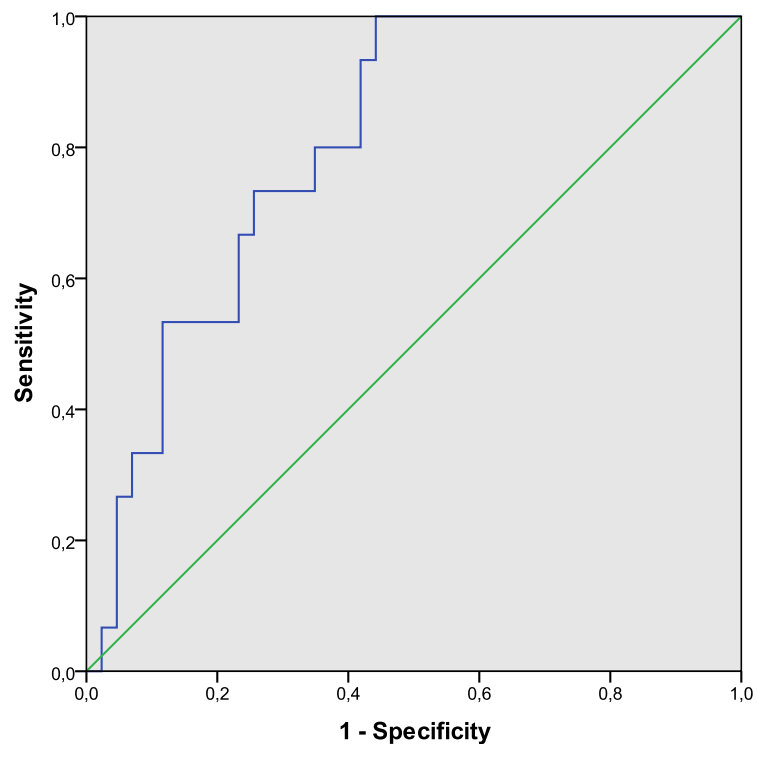

Fig. 1. ROC Curve using TTL variable as predictor of NSLN metastases. 
questioned. This trial indicated that ALND has no significant impact on either disease-free or overall survival of patients with SLN metastases, but these results are only applicable to patients who met the study inclusion criteria (fewer than three SLN metastases, treatment with breast-conserving surgery, radiation therapy and systemic therapy) [6].

In patients for whom the results of the Z0011 are not applicable, prediction models for NSLN metastases are still helpful for ALND surgical decisions. The current predictor model using TTL can be intraoperatively valuable, as a simple, fast and accurate method of assessing the probability of NSLN.

Our study has the limitations of being retrospective with a small sample size. The clinical implications of TTL in how we surgically manage patients are therefore limited. Larger prospective studies are needed to determine the prognostic implications.

\section{Ethical approval}

Ethical approval was not required for this review.

\section{Funding source}

This research did not receive any specific grant from funding agencies in the public, commercial, or not-for-profit sectors.

\section{Conflict of interest statement}

None declared.

\section{Acknowledgements}

We would like to thank Ana Correia, Inês Morujão and Carlos Vitorino for providing helpful support and suggestions during the research.

\section{References}

[1] Veronesi U, Paganelli G, Galimberti V, Viale G, Zurrida S, Bedoni M, et al. Sentinel-node biopsy to avoid axillary dissection in breast cancer with clinically negative lymph-nodes. Lancet Lond Engl 1997 Jun 28;349(9069): 1864-7.

[2] Banerjee SM, Michalopoulos NV, Williams NR, Davidson T, El Sheikh S, McDermott $\mathrm{N}$, et al. Detailed evaluation of one step nucleic acid (OSNA) molecular assay for intra-operative diagnosis of sentinel lymph node metastasis and prediction of non-sentinel nodal involvement: experience from a London Teaching Hospital. Breast 2014 Aug:23(4):378-84.

[3] Heilmann T, Mathiak M, Hofmann J, Mundhenke C, van Mackelenbergh M, Alkatout I, et al. Intra-operative use of one-step nucleic acid amplification (OSNA) for detection of the tumor load of sentinel lymph nodes in breast cancer patients. J Cancer Res Clin Oncol 2013 Oct;139(10):1649-55.

[4] Chaudhry A, Williams S, Cook J, Jenkins M, Sohail M, Calder C, et al. The realtime intra-operative evaluation of sentinel lymph nodes in breast cancer patients using One Step Nucleic Acid Amplification (OSNA) and implications for clinical decision-making. Eur J Surg Oncol EJSO 2014 Feb;40(2):150-7.

[5] Tiernan JP, Verghese ET, Nair A, Pathak S, Kim B, White J, et al. Systematic review and meta-analysis of cytokeratin 19-based one-step nucleic acid amplification versus histopathology for sentinel lymph node assessment in breast cancer: sentinel lymph node assessment in breast cancer. Br J Surg 2014 Mar;101(4):298-306.

[6] Lyman GH, Temin S, Edge SB, Newman LA, Turner RR, Weaver DL, et al Sentinel lymph node biopsy for patients with early-stage breast cancer: american society of clinical Oncology clinical practice guideline update. J Clin Oncol 2014 May 1:32(13):1365-83.

[7] Ohi Y, Umekita Y, Sagara Y, Rai Y, Yotsumoto D, Matsukata A, et al. Whole sentinel lymph node analysis by a molecular assay predicts axillary node status in breast cancer. Br J Cancer 2012 Oct 9;107(8):1239-43.

[8] Osako T, Iwase T, Kimura K, Horii R, Akiyama F. Sentinel node tumour burden quantified based on cytokeratin 19 mRNA copy number predicts non-sentinel node metastases in breast cancer: molecular whole-node analysis of all removed nodes. Eur J Cancer 2013 Apr;49(6):1187-95.

[9] Espinosa-Bravo M, Sansano I, Pérez-Hoyos S, Ramos M, Sancho M, Xercavins J, et al. Prediction of non-sentinel lymph node metastasis in early breast cancer by assessing total tumoral load in the sentinel lymph node by molecular assay. Eur J Surg Oncol EJSO 2013 Jul;39(7):766-73.

[10] Peg V, Espinosa-Bravo M, Vieites B, Vilardell F, Antúnez JR, de Salas MS, et al. Intraoperative molecular analysis of total tumor load in sentinel lymph node: a new predictor of axillary status in early breast cancer patients. Breast Cancer Res Treat 2013 May;139(1):87-93. 